\title{
Technique for fabricating complex structured fibers by rolling of glass preforms
}

\author{
Zheng Gang Lian*, John A. Tucknott, Nicholas White, Limin Xiao, Xian Feng, David N Payne \\ and Wei H. Loh \\ Optoelectronics Research Centre, University of Southampton, Southampton SO17 1BJ, United Kingdom \\ *Corresponding author: zl4e09@orc.soton.ac.uk
}

\begin{abstract}
We propose a new technique for making optical fiber preforms in order to realize complex structured optical fibers. The preforms are fabricated by rolling up custom-patterned flat glass sheets, and the resulting cylindrical structure drawn into optical fibers. OCIS Codes: (060.2280) Fiber design and fabrication; (060.4005) Microstructured fibers
\end{abstract}

\section{Introduction}

The last decade has seen a surge of intense interest in the design and application of complex optical fiber structures extending beyond conventional core-clad fibers. While much of this has been driven by the advent of photonic crystal fibers [1], developments in the realization of Bragg fibers [2] and optoelectronic fibers [3] also highlight the rich potential that can be unlocked from considering complex fiber designs. The emergence of these fibers has already given rise to a range of new applications, such as efficient broadband (supercontinuum) generation [4] of light with spectral widths spanning over $2 \mu \mathrm{m}$, and high power infrared transmission [2]. The most common technique for fabricating microstructured optical fibers is the stack-and-raw method, namely, assemble a large number of hollow glass tubes (or capillaries) together to form the preform, and then draw the combined structure into a fiber. However, the need to prepare and stack a large number of tubes together can be very time-consuming to properly assemble in order to achieve the desired optical characteristic, due to the sensitivity of, e.g. the dispersion, on the relative position of the holes. Alternate techniques used to realize microstructured fibers include drilling the holes in a solid glass preform [5], or extruding glass [6] through an appropriately machined die. Although drilling appears straightforward, the drilling of deep holes in glass is also a time-consuming operation. Glass extrusion is a promising but complex technique; it requires very good control of the glass temperature and pressure to obtain the needed uniformly throughout the extrusion process, and currently takes several hours to extrude a high quality piece $30 \mathrm{~cm}$ or longer.

We present here a new method for fabricating complex glass fiber structures that has some potentially very interesting advantages. The approach can enable rapid fabrication of quite general microstructured fibers as well as Bragg and optoelectronic fiber structures; more intriguing, it will be able to incorporate additional features into the preform which are difficult to accomplish by previous methods, such as helical and/or longitudinally varying features in the preform. This new technique is by rolling up pre-patterned glass sheets into fiber preforms.

\section{Concept}

The basic concept behind the glass rolling technique is quite simple. By creating a pattern on a planar glass sheet and then rolling the sheet up into a cylinder (not dissimilar to that of a Swiss roll), we would arrive at a structured glass preform. As planar fabrication techniques are already well developed, our approach thus relies on relatively mature fabrication processes except one - the glass rolling step, which is what we demonstrate in this work. Compared with previously mentioned techniques, this approach could be fast, with the ability to produce high quality features. Thus, for example, the patterning could be accomplished directly with precision glass dicing saws - already used to mechanically create ridge waveguides in LiNbO3 [7] - to cut grooves in the glass sheet in a matter of minutes.

Fig. 1 shows schematically the approach to fabricate a microstructured fiber preform from a glass sheet. In Fig. 1(a), a rectangular glass sheet is ready for cutting and rolling. Grooves are cut using dicing saws on the top surface of the sheet (Fig. 1(b)). The depth and spacing between neighbor grooves can be varied. The width of the grooves can also be variable, depending e.g. on the thickness of the saw blade. After the grooves are cut, the glass sheet is heated and rolled as shown in Fig. 1(c) and (d) to form a cylinder. It is clear that the grooves in the original flat glass sheet are now located at different layers within the cylinder, and act as air holes in the rolled fiber preform. The entire rolling process typically takes no more than a few minutes to complete, which could make this approach particularly attractive as a rapid prototyping technique for testing novel microstructured fiber designs. 
(a)

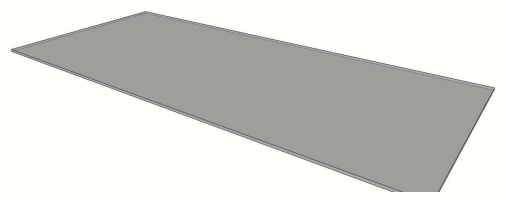

(c)

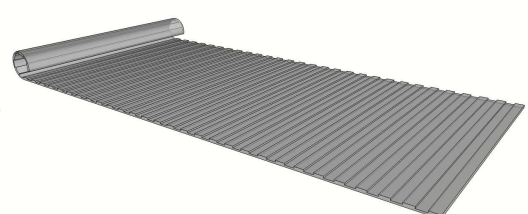

(b)

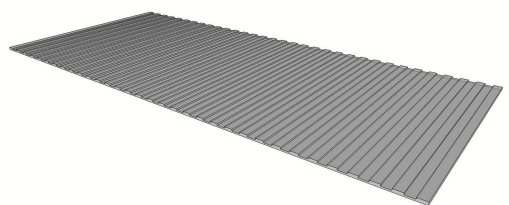

(d)

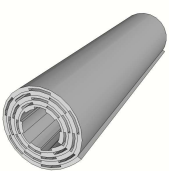

Fig. 1 Schematic diagrams of the glass sheet rolling process for making fiber preforms. (a) A flat glass sheet; (b) grooves were cut on the surface of the sheet; (c) the cut glass sheet is heated and rolled to form a cylinder and (d) the rolled structured preform is obtained, with the cut grooves forming as holes located between the glass layers.

\section{Experiment and results}

The glass we used to develop the process is borosilicate (Borofloat ${ }^{\circledR 3} 3$ ). Although the optical loss at $1550 \mathrm{~nm}$ of the Borofloat glass is quite high $(12 \mathrm{~dB} / \mathrm{m})$, it is an affordable commercial glass and readily available in reasonable sized sheets. Typical dimensions of the Borofloat glass sheets used for rolling were $200 \mathrm{~mm} \times 90 \mathrm{~mm} \times 1.1 \mathrm{~mm}$. Grooves were cut in the surface of the Borofloat glass sheet with a dicing saw. The thickness of the saw blade was $1 \mathrm{~mm}$; the grooves are therefore $1 \mathrm{~mm}$ wide as shown in Fig. 2(a) and (b). The spacing between adjacent grooves was $2 \mathrm{~mm}$ (centre to centre), which will yield a pitch of $2 \mathrm{~mm}$ in structured preform. The depth of the grooves can be varied, with deeper cuts resulting in larger holes in the final fiber structure. The depth of the grooves here employed was $0.45 \pm 0.05 \mathrm{~mm}$.

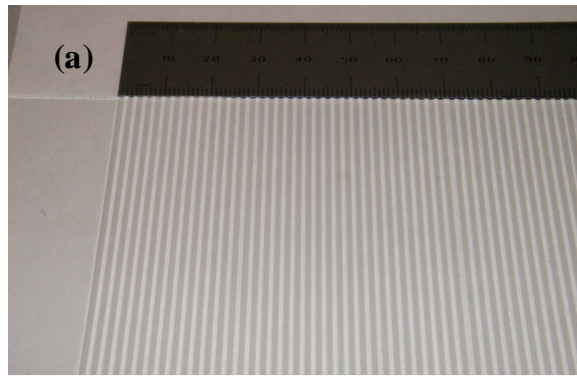

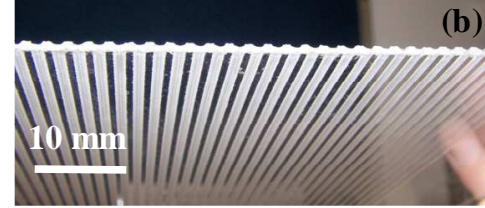

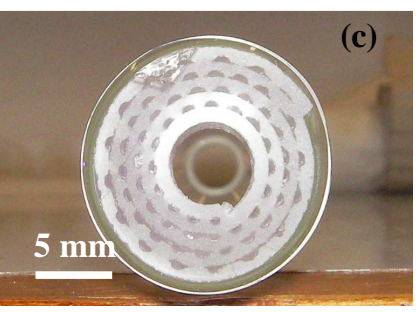

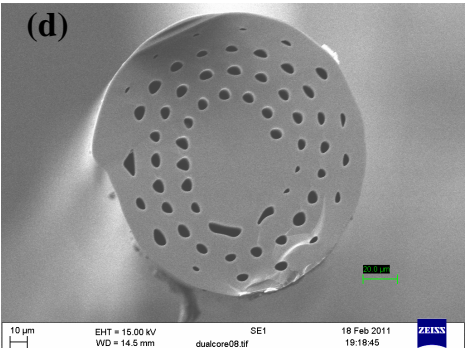

$\stackrel{10 \mathrm{~mm}}{\mapsto}$

Fig. 2 (a) Top view of grooves cut on the surface of the Borofloat sheet. The width of the grooves was $1 \mathrm{~mm}$ and the spacing between neighbor grooves was $2 \mathrm{~mm}$; (b) Side view of grooves on the Borofloat sheet; (c) cross-sectional view of the rolled Borofloat preform; (d) SEM image of the cross section of the fiber drawn from the rolled preform. The fiber diameter is $150 \mu \mathrm{m}$.

The glass sheet rolling process was carried out in a resistance furnace. A pyrophyllite sheet was installed at the bottom of the furnace, upon which the Borofloat glass sheet was placed, with the grooves facing upwards. A $6 \mathrm{~mm}$ diameter 'rolling pin' made from glass sealing alloy was used as a mandrel for rolling the glass sheet. Glass sealing alloy was chosen here because it has a thermal expansion coefficient $\left(4.81 \times 10^{-6} \mathrm{~K}^{-1}\right.$ at $\left.20-200^{\circ} \mathrm{C}\right)$ close to that of the Borofloat glass $\left(3.25 \times 10^{-6} \mathrm{~K}^{-11}\right.$ at $\left.20-300^{\circ} \mathrm{C}\right)[8]$. The optimum temperature we found for the rolling was $740^{\circ} \mathrm{C}$, 
which is lower than the Borofloat glass softening temperature of $820^{\circ} \mathrm{C}$ [8]. The rolling process itself took just 5 minutes. After rolling, the temperature was decreased to $525^{\circ} \mathrm{C}$ (the $\mathrm{T}_{\mathrm{g}}$ of Borofloat ${ }^{3} 33$ ) for annealing ( 1 hour) and then brought down to room temperature. Fig. 2(c) shows the cross-sectional view of the rolled preform. The mandrel has been removed, accounting for the $6 \mathrm{~mm}$ hole in the centre. The outer diameter of the preform is $16 \mathrm{~mm}$. The grooves on the surface of the glass sheet shown in Fig 2(a) and (b) were well maintained in the cylindrical structure. More layers to the preform can be obtained from using longer glass sheets or stacking multiple glass sheets.

The rolled preform was pulled into fiber with different diameters, from $90 \mu \mathrm{m}$ to $200 \mu \mathrm{m}$. A vacuum was applied to the central hole during the fiber drawing to collapse it, to form a solid core. Fig. 2(d) is a Scanning Electron Microscope (SEM) image of the cross section of a $150 \mu \mathrm{m}$ diameter fiber drawn from the rolled preform. The solid core was slightly oval because the central hole in the preform did not symmetrically collapse during the fiber drawing in this case. We note, however, that the interfaces between the rolled layers have sealed up well, leaving the grooves to form holes in the fiber structure as intended.

Although the result described above was for a $1 \mathrm{~mm}$ thick glass sheet, we have investigated the rolling process for sheet thicknesses from $0.7 \mathrm{~mm}$ to $2 \mathrm{~mm}$, and all these sheets have also been rolled successfully into cylindrical structures. It is not difficult to see that for a fixed sheet length, a different glass thickness will result in a change to the diameter of, and the size of the holes achievable in, the resulting preform.

\section{Conclusion}

A glass sheet rolling technique has been demonstrated for fabricating structured fiber preforms. Microstructured fibers were drawn from the preform, with the desired microstructures maintained in the fiber. Although the demonstration here is with grooves cut into the glass sheet in the direction perpendicular to the direction of the rolling to show a 'conventional' microstructured preform, it should be evident that more unusual structures can be obtained with this technique, e.g. with the grooves cut at an angle to the rolling direction, a spiral structure will be encapsulated in the cylindrical preform. In effect, the rolling process can be viewed as a mapping from a 2dimensional (planar) structure to a 3-dimensional structure. As arbitrary planar patterns can be easily fabricated on a glass sheet, e.g. through standard lithography and etch processes, these results should be of interest for the fabrication of a wide range of complex glass fiber structures, including photonic crystal fibers, Bragg fibers, optoelectronic fibers, nonperiodic fiber structures, longitudinally varying fibers, etc.

We gratefully acknowledge support from the Engineering and Physical Sciences Research Council for funding of this work, under the EPSRC Centre for Innovative Manufacturing in Photonics, and E. Weatherby for his skillful machining of many of the parts used in creating the prototype glass rolling rig.

\section{References}

1. Cregan, R.F., B.J. Mangan, J.C. Knight, T.A. Birks, S.S.J. Russell, R.J. Roberts, and D.C. Allan, Single-mode photonic band gap guidance of light in air. Science, 1999. 285: p. 1537-1539.

2. Temelkuran, B., S.D. Hart, G. Benoit, J.D. Joannopoulos, and Y. Fink, Wavelength-scalable hollow optical fibres with large photonic bandgaps for $\mathrm{CO}_{2}$ laser transmission. Letters to Nature, 2002. 420: p. 650-653.

3. Bayindir, M., F. Sirin, A.F. Abouraddy, J. Viens, S.D. Hart, J.D. Joannapoulos, and Y. Fink, Metal-insulator-semiconductor optoelectronic fibres. Letters to Nature, 2004. 431: p. 826-829.

4. Ranka, J.K., R.S. Windeler, and A.J. Stentz, Visible contunuum generation in air-silica microstructure optical fibers with anomalous dispersion at $800 \mathrm{~nm}$. Optics Letters, 2000. 25(1): p. 25-27.

5. Knight, J.C., T.A. Birks, P.S.J. Russell, and D.M. Atkin, All-silica single-mode optical fiber with phtonic crystal cladding. Optics Letters, 1996. 21(19): p. 1547-1549.

6. Feng, X., T.M. Monro, V. Finazzi, R.C. Moore, K. Frampton, P. Petropoulos, and D.J. Richardson, Extruded singlemode, high nonlinearity tellurite glass holey fibre. Electronic Letters, 2005. 41(15): p. 835-837.

7. Kurimura, S., Y. Kato, M. Maruyama, Y. Usui, and H. Nakajima, Quasi-phase-matched adhered ridge waveguide in LiNbO $\mathrm{H}_{3}$. Applied Physics Letters, 2006. 89: p. 191123-1-3.

8. Schott Borofloat 33 product information, Schott: Jena, Germany. 